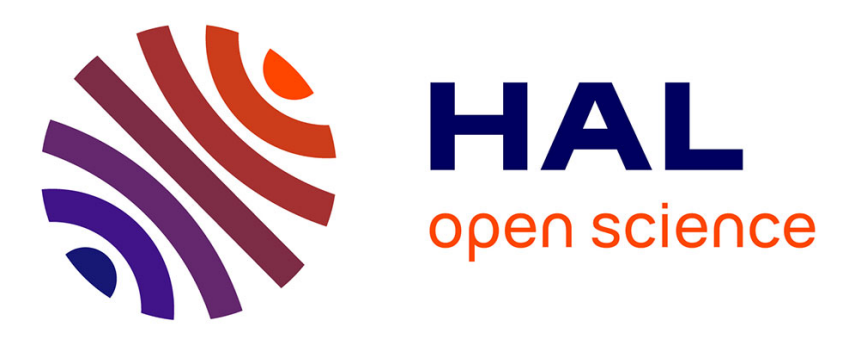

\title{
Bayesian based fault diagnosis : application to an electrical motor
}

Amine Mechraoui, Kamal Medjaher, Noureddine Zerhouni

\section{To cite this version:}

Amine Mechraoui, Kamal Medjaher, Noureddine Zerhouni. Bayesian based fault diagnosis: application to an electrical motor. IFAC WC 2008 - 17th IFAC World Congress, Jul 2008, Séoul, South Korea. 6 p. hal-00298240

\section{HAL Id: hal-00298240 \\ https://hal.science/hal-00298240}

Submitted on 16 Jul 2008

HAL is a multi-disciplinary open access archive for the deposit and dissemination of scientific research documents, whether they are published or not. The documents may come from teaching and research institutions in France or abroad, or from public or private research centers.
L'archive ouverte pluridisciplinaire HAL, est destinée au dépôt et à la diffusion de documents scientifiques de niveau recherche, publiés ou non, émanant des établissements d'enseignement et de recherche français ou étrangers, des laboratoires publics ou privés. 


\title{
Bayesian based fault diagnosis: application to an electrical motor
}

\author{
A. Mechraoui ${ }^{*}$, K. Medjaher ${ }^{* *}$, N. Zerhouni ${ }^{* *}$ \\ * GIPSA-lab, CNRS UMR 5216, ENSIEG, Domaine Universitaire \\ BP46, 38402 Saint Martin d'Hères cedex, France \\ ** FEMTO-ST Institute, UMR CNRS 6174 - UFC/ENSMM/UTBM, \\ Automatic Control and Micro-Mechatronic Systems Department \\ 24, rue Alain Savary, 25000 Besançon, France \\ (e-mail:kamal.medjaher@ens2m.fr)
}

\begin{abstract}
In the literature, several fault diagnosis methods, qualitative as well as quantitative, are proposed. The main objective of these methods is in one hand, to allow detection, isolation and identification of faults; and in the other hand to insure safety, reliability and availability of systems. This paper presents a diagnosis method based on the use of a new and suitable mathematical tool: bayesian networks. Their learning and inference capabilities allow to model complex processes by taking into account the uncertainty and the incompleteness of the provided knowledge. Furthermore, the graphical representation of causal relations existing between variables, events or physical phenomena makes bayesian networks easy to use and leads to models which can be understandable by even a non specialist of the modeled domain.
\end{abstract}

Keywords: Diagnosis, Fault isolation, Bayesian networks, Inference, Probabilities

\section{INTRODUCTION}

Fault diagnosis consists in isolating and identifying the causes of an abnormal operation of a given system. This abnormal situation can be expressed (or characterized) by a set of symptoms which are incoherences between the observed behavior and the nominal behavior of the process. Several diagnosis methods have been proposed in the literature. These methods can be classified into two main categories: those using an analytical model and those which don't use an analytical model. The first ones (which include parity space, parameter estimation, state observers, etc.) $[1,2]$ can be used in the case where the process for which one aims to perform a fault diagnosis is sufficiently known so that we can derive a model that reflects as faithfully as possible its dynamic behavior. The derived model is then used to generate what is called fault indicators (analytical redundancy relations, residuals, etc.). The online evaluation and analysis of these indicators allow to detect and to isolate faults that can affect the process. However, in practice, systems or processes are often complex and involve several energy domains. Therefore, the model is either difficult (or even impossible) to obtain, or unexploitable because of its complexity (nonlinear model, presence of loops, etc.). The second category of diagnosis methods [3, 4], which don't use an analytical model and which are generally derived from artificial intelligence techniques (neural networks, expert systems, case based reasoning, etc.), can be used instead of model based methods in cases where the model does not exist or difficult to obtain. However, these methods, called also qualitative methods, need a rich database (experience feedback data or experimental data for instance) to perform a good learning; task which can be difficult to satisfy (e.g. case of systems in design stage or newly put in service). Furthermore, in practice the knowledge one has about the system can be incomplete or uncertain. Thus, the use of a mathematical tool introducing the notion of probability to take into account this uncertainty and/or incompleteness can be a convenient solution. In the present contribution, we have used bayesian networks for the possibilities they offer in modeling of complex and stochastic systems and also for their learning and inference capabilities [5]. Compared to the previous referenced approaches, bayesian networks allow graphical representation of the knowledge under its different types (rules, causal relationships, experts' statements, physical laws, etc.). In addition, parameter as well as graphical structure update is easy to perform when using this kind of tool [5]. In this paper, bayesian networks are used to model the knowledge we have about the process and to perform a fault diagnosis. The tool's qualitative aspect (directed acyclic graph) allows to represent graphically the causal relations between the process variables. The quantitative part of bayesian networks tool consists in determining the a priori and conditional probability tables of each variable in the generated graph. These probabilities can be given by an expert of the process or obtained by a learning method or algorithm from an experimental or experience feedback database. In the literature, many research works have been proposed on bayesian networks but, most of them are focused on learning algorithms that allow to construct the graphical model and estimate the probabilities of each node of the derived graph [6]. In this contribution, the diagnosis task consisted in computing the a posteriori probability of each process component (or node) given a set of new observations (also called evidences).

The present paper is organized as follows: the second sec- 
tion presents a brief description of the bayesian networks tool. This description is illustrated by a simple example of fault diagnosis on a laptop. In the third section, the proposed approach is applied to diagnose faults on a permanent magnet synchronous motor. For this application, several scenarios are simulated and the results obtained are discussed. Finally, a conclusion is given in section four.

\section{BAYESIAN NETWORKS}

\subsection{Definition}

A bayesian network is defined by:

- a directed acyclic graph $G, G=(V, E)$, where $V$ is the set of nodes of $G$, and $E$ is the set of edges of $G$;

- a finite probabilised space $(\Omega, Z, P)$;

- a set of random variables associated to the nodes of the graph and defined on $(\Omega, Z, P)$, such that:

$$
P\left(V_{1}, V_{2}, \ldots, V_{n}\right)=\prod_{i=1}^{n} P\left(V_{i} \mid C\left(V_{i}\right)\right.
$$

where $C\left(V_{i}\right)$ is the set of causes (parents) of $V_{i}$ on the graph $G$.

In other words, bayesian networks provide a formalism to represent a joint probability distribution on a set of random variables. Bayesian networks can be considered as a convenient tool allowing to handle two big problems commonly encountered in artificial intelligence, in applied mathematics and in engineering: uncertainty and complexity. Bayesian networks are a combination result between probability theory and graph theory. They are thus:

- models for representing knowledge,

- and machines for computing conditional probabilities.

Modeling by using bayesian networks is performed in two steps: the qualitative step (construction of the network or the graph) and the quantitative step (deriving or estimating the probability distribution tables).

\subsection{Modeling}

Qualitative step: this step allows to derive the graphical structure of the bayesian network that represents the causal relations between the different variables of the process under study. This structure can be obtained by two different ways: by exploiting the experts' knowledge of the process or, by using a well documented database (learning). To illustrate how this step is performed, we consider a simple example through which we wish to model a dysfunction affecting a useful working tool that became more than indispensable for a researcher: a laptop.

After pushing the power button, the researcher notes that his/her laptop does not start. So, two possible causes of this dysfunction come to his/her mind: the problem can come from the battery which is empty or, from the mother board which is faulty. For simplicity of the model, we suppose that the main supply from an AC plug is not considered in this example. Moreover, we consider that the battery has a level indicator which allows to know at each moment if it is loaded or not.

The first step (qualitative) in constructing the bayesian network of the previous described abnormal situation consists in a graphical representation of the causal relations between the four variables (or nodes) associated to the following events or elements: S (doesn't Start: true, false), B (Battery: loaded, not loaded), I (level Indicator: full, half, empty) and M (Mother board: ok, degraded). The derived graph corresponding to the intuitive analysis of the researcher is given in figure 1 .

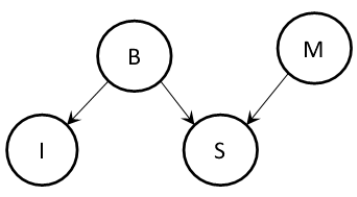

Fig. 1. Starting problem of a laptop (qualitative step)

To isolate the cause of the abnormal operation (the laptop does not start), the researcher decides to have a look on the level indicator of the battery and notes that this one indicates the middle position. The researcher concludes then that the problem would be, more probably, caused by the mother board (degraded). Thus, the fact that I indicates that the battery is half loaded strengthens the belief on $\mathrm{M}$ as the most probable cause of the observed dysfunction. From the general point of view, the sure (or certain) information (which is sometimes called evidence or observation), like the fact that the laptop doesn't start, propagates on the bayesian network by modifying the beliefs one had before on the facts. The recent observed information is then propagated on the graph leading to an update (recompute) of the nodes' probabilities.

Quantitative step: it consists in associating to each node a probability table (definition of all the probabilities of a variable or a node for each one of its possibles values (modalities) knowing the values of its parents (causes of the node)).

To explain this quantitative aspect of bayesian networks, let's use again the previous described example related to a laptop starting problem (see figure 1). We will assimilate the belief or probability of a fact to a mathematical probability and we will show the similarity between the qualitative results obtained previously and the quantitative ones obtained hereafter.

It is supposed that the a priori marginal probabilities $P(M)$ and $P(B)$ are obtained by experience on the system (practically, these probabilities are given by an expert of the modeled domain or learned from a rich database). For instance, if we know that the mother board breaks down in $5 \%$ of cases, then one has a probability of 0.05 . And, if we know that the researcher forgets to load the battery of his/her laptop before it is totaly empty in $10 \%$ of cases then the probability for the battery to be empty is 0.1 .

For the a priori conditional probability $P(S \mid B, M)$, we consider the values given in the following table.

\begin{tabular}{|c|c|c|c|c|}
\hline$M$ & \multicolumn{2}{|c|}{ degraded } & \multicolumn{2}{c|}{ ok } \\
\hline$B$ & loaded & not loaded & loaded & not loaded \\
\hline$P(S=$ true $\mid M, B)$ & 0.9 & 1 & 0.05 & 1 \\
\hline$P(S=$ false $\mid M, B)$ & 0.1 & 0 & 0.95 & 0 \\
\hline
\end{tabular}

For example, it is supposed that in the case where the mother board is out of service or degraded ( $M=$ degraded) and the battery is loaded ( $\mathrm{B}=$ loaded) the probability that the laptop does not start is equal to 0.9. Likewise, if the battery is completely empty then, we know that the laptop 
will not start independently of the state of the mother board; the associated probability is equal to 1 (remind that the main supply is not considered in this example). It remains the case where the mother board works correctly $(\mathrm{M}=\mathrm{ok})$ and the battery is not empty $(\mathrm{B}=$ loaded $)$. For this configuration, one supposes that there is $95 \%$ of chance that the laptop starts correctly (the remaining 5\% are caused by other events which are not taken into account in the coming calculations).

For the probability $P(I \mid B)$, we suppose that the researcher often forgets to completely load the battery of his/her laptop. We consider then a probability of $25 \%$ for the battery to be completely loaded knowing that it is not empty, and a probability of $75 \%$ for the battery to be half loaded in the same case. And finally, we are sure that the battery is empty knowing that it has not been loaded before. Now, in the case where the laptop does not start, which one of the two conditional causes (the mother board is degraded or the battery is empty) is the most probable? This question can be mathematically answered by using probability calculations detailed hereafter.

Probability calculations: in the example given in figure 1 , one would like to know the probability $P(B, M \mid S=$ true). This probability can be obtained by using the basic probability formulas. The main formula expressing the relation between the conditional probability and the joint probability is given by the following equation:

$$
P(a, b)=P(a \mid b) P(b)=P(b \mid a) P(a) .
$$

Equation (2) leads to the well known Bayes formula:

$$
P(a \mid b)=\frac{P(b \mid a) P(a)}{P(b)},
$$

which can be expressed under the following global form:

$$
P(a \mid b, c)=\frac{P(b \mid a, c) P(a \mid c)}{P(b \mid c)} .
$$

In addition to these given relations, one needs the marginal law used to calculate the marginal probability on a bayesian network:

$$
P(A)=\sum_{B} P(A, B) .
$$

Note that for each bayesian network is associated a universe $U$ represented by the joint probability distribution $P(U)$. This probability is obtained by the multiplication of all the a priori marginal probabilities (for nodes without parents) and the conditional probabilities (for nodes with parents) (Eq. (1)). By using the previous relations, one can compute the probability $P(B, M \mid S=$ true $)$. According to equation (1), we can write:

$$
P(M, B, S, I)=P(M) P(B) P(S \mid M, B) P(I \mid B) .
$$

The achieved calculations lead then to the results presented in table 1.

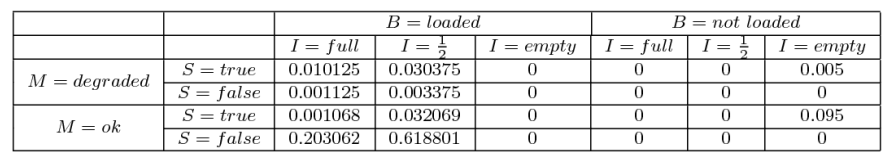

Table 1. Joint probability table

By using the results given in table 1 , one can calculate any desired probability. The universe $P(M, S, B, I)$ contains the desired probability $P(B, M \mid S)$, which can be derived from Eq.(2) and Eq.(5):

$$
P(B, M \mid S)=\frac{P(B, S, M)}{P(S)}
$$

with

$$
P(S)=\sum_{B, M, I} P(M, B, S, I)
$$

and

$$
P(B, M, S)=\sum_{I} P(B, M, S, I) .
$$

The results obtained by using Eq. (7) are given by the following table. From this latter, one can also calculate the

\begin{tabular}{|c|c|c|c|c|}
\hline$M$ & \multicolumn{2}{|c|}{ degraded } & \multicolumn{2}{c|}{ ok } \\
\hline$B$ & loaded & not loaded & loaded & not loaded \\
\hline$P(M, B \mid S=$ true $)$ & 0.2210 & 0.027285 & 0.233287 & 0.518417 \\
\hline$P(M, B \mid S=$ false $)$ & 0.00551 & 0 & 0.99449 & 0 \\
\hline
\end{tabular}

probabilities $P(M \mid S)$ and $P(B \mid S)$ by using the marginal probability law:

\begin{tabular}{|c|c|c|}
\hline$M$ & degraded & ok \\
\hline$P(M \mid S=$ true $)$ & 0.248285 & 0.751704 \\
\hline$P(M \mid S=$ false $)$ & 0.00551 & 0.99449 \\
\hline$B$ & loaded & not loaded \\
\hline$P(B \mid S=$ true $)$ & 0.454287 & 0.545702 \\
\hline$P(B \mid S=$ false $)$ & 1 & 0 \\
\hline
\end{tabular}

$$
P(M \mid S)=\sum_{B} P(B, M \mid S)
$$

and

$$
P(B \mid S)=\sum_{M} P(B, M \mid S)
$$

The two following probability tables present the obtained results.

According to the two last tables we note that in the case where the laptop doesn't start, $S=$ true, one has tendency to think that the battery is not loaded with a probability equal to $0.5457(P(B=$ not loaded $\mid S=$ true $)=0.5457)$. But, after verifying the level indicator position, we observe that this latter is at the middle position (the battery is half loaded). This new information will then change our belief on the fact that the battery was the cause of the abnormal operation (dysfunction) of the laptop (doesn't start). The probability calculation $P\left(M, B \mid S=\right.$ true, $\left.I=\frac{1}{2}\right)$ will allow to diagnose the most probable cause of the observed problem.

$$
P\left(M, B \mid S=\text { true }, I=\frac{1}{2}\right)=\frac{P(M, B, S, I)}{\sum_{M, B} P\left(M, B, S=\text { true }, I=\frac{1}{2}\right)}
$$

The achieved calculations lead to the results given in the following table:

\begin{tabular}{|c|c|c|c|c|}
\hline $\mathrm{M}$ & \multicolumn{2}{|c|}{ degraded } & \multicolumn{2}{c|}{ ok } \\
\hline $\mathrm{B}$ & loaded & not loaded & loaded & not loaded \\
\hline$P\left(M, B \mid S=\right.$ true,$\left.I=\frac{1}{2}\right)$ & 0.904337 & 0 & 0.095477 & 0 \\
\hline
\end{tabular}

In other words, in the case where the level indicator shows that the battery is half loaded, the abnormal operation (dysfunction of the laptop) would have been most probably due to the mother board which is degraded (with a probability equal to 0.9 ). This confirms the conclusions of the intuitive reasoning performed during the qualitative step. 


\subsection{Bayesian inference}

The probabilist inference, also called update of probabilities, corresponds to any calculation related to a probability distribution associated to a bayesian network [7]. The problem of computing the a posteriori probabilities on a bayesian network is NP-difficult [8]. However, to overcome this difficulty several algorithms are proposed in the literature.

The first exact algorithms related to bayesian inference have been proposed in [7]. They were based on a message passing architecture and were limited to graphs in form of trees. In these kind of algorithms, at each node is associated a processor which sends messages asynchronously to its neighbors until an equilibrium is reached on a finite number of steps. This method (or algorithm) has been extended to any type of network and led to what is called JLO algorithm. This latter, also called junction tree algorithm, is well developed in [9] where a method is proposed to transform any kind of network to a tree in order to facilitate the inference.

Though the inference in any network is NP-difficult, The time consuming of each method previously mentioned is computable in advance. Thus, when the result exceeds a reasonable limit, one prefers to use an approximative method or algorithm instead of an exact one [7]. These approximative methods exploit the topology of the network and perform a sampling on local subsets of variables in a sequential and concurrent way [10].

Other methods have been proposed in the literature like that one of Shafer-Shenoy [11] or the symbolic probabilist inference proposed by D'Ambrosio [12]. All these methods have been since studied by Zhang and Poole and a comparison between them has been performed to underline the advantages and the drawbacks of each one of them [13].

\section{APPLICATION : FAULT DIAGNOSIS ON AN ELECTRICAL MOTOR}

In this part, bayesian networks are used as a tool for diagnosing faults on an electrical system. We will try to apply the approach detailed in the previous sections on a permanent magnet synchronous motor and we will focus our study on a specific function of the system: the generation by the rotor of a rotating torque.

Remark: the original application is in fact a new generation of a real permanent magnet synchronous motor designed and developed by a well known French train manufacturer. For confidentiality reasons, we are not allowed to publish any document related to the system. Instead, we have replaced the studied system by a generic permanent magnet synchronous motor.

\subsection{System description}

A permanent magnet synchronous motor (see figure 2) is mainly composed of a rotor and a stator that generate a rotation motion transmitted by a transmission shaft to a load. The stator is supplied by a three-phase signal and creates a rotating field. Thanks to magnets, the rotor rotates at the same speed than that one of the rotating field created by the stator.

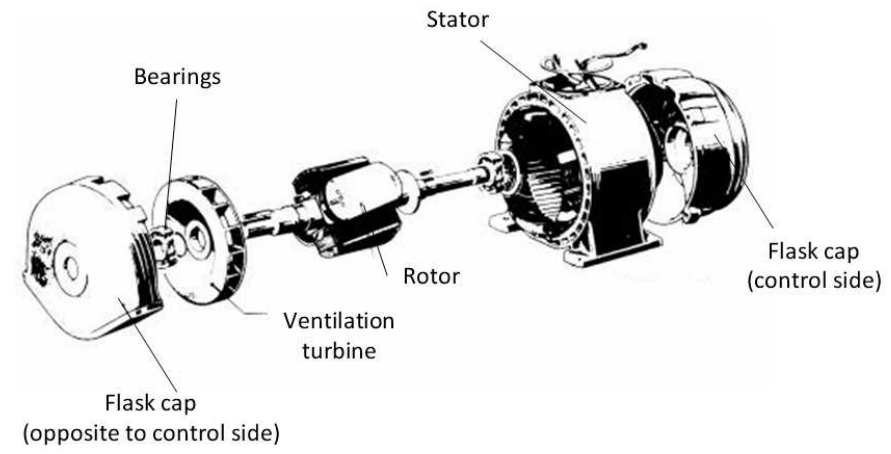

Fig. 2. Permanent magnet synchronous motor

The main objective of this application is to perform a diagnosis on the rotation motion of the motor's shaft (we will focus on the availability or not of the rotation motion).

\subsection{Modeling}

To build the bayesian network used in the diagnosis step of the motor, we have used a database, given by the expert of the system, which includes a functional decomposition of the entire electrical motor. From this database, one can easily define all the causal relations existing between the different nodes (representing the components) of the graph (qualitative step) and then estimate the conditional probability tables related to each node (quantitative step).

Qualitative step: the available functional decomposition of the motor is used hereafter in order to identify the different nodes of the corresponding graphical model and the causal relations existing between them. In the current bayesian network, three types of nodes which allow to achieve the diagnosis are introduced: the nodes related to components of the motor, the action nodes (don't confuse with action nodes used in some softwares) and the monitored nodes (for which the state can be observed by sensors or any other observation mean). After identifying the nodes, the modes corresponding to each node and the causal relations between the different variables or nodes (qualitative step); we have proceeded to the construction of the bayesian network used in the diagnosis of possible faults that can affect the components involved in the rotation motion of the motor's shaft (see figure 3 ).

Quantitative step: this consists in estimating the a priori marginal and conditional probabilities of each node of the network. This estimation is obtained from the knowledge provided by the expert of the motor. We have questioned the expert by asking him to position his expertise on a probability scale as shown in figure 4 (more details on this method are given in reference [5]). The expert's appreciations are then transposed to numerical values corresponding to probabilities of occurrence of events. These values can then be modified (or updated) according to simulation or experimental results. The number of estimated probabilities depends particularly on the number of nodes and on the number of modes associated to each one of the nodes. In our case, we have estimated this number to 292 probabilities.

The calculations on the bayesian network of figure 3 are manually tedious to do. To overcome this difficulty, 


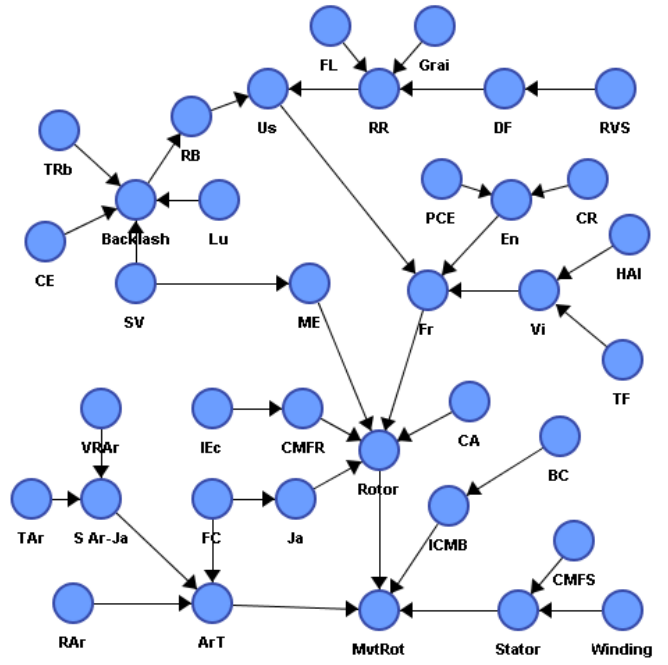

Fig. 3. Bayesian network used for fault diagnosis

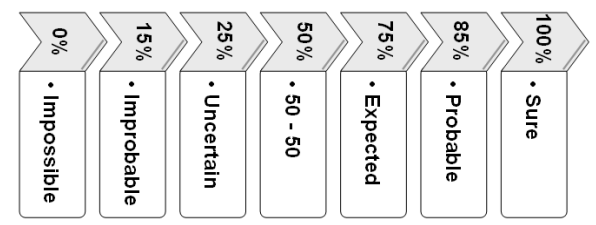

Fig. 4. Scale used for an a priori probability estimation we have used a software called BayesiaLab in order to compute the joint, marginal and conditional probabilities. BayesiaLab is a suitable software which can be used to model, learn and analyze bayesian networks. However, there exists a large choice of other softwares and toolboxes that deal with bayesian networks (BNT : Bayesian Networks Toolbox, Hugin, NETICA, etc.) and which can be used successfully.

\subsection{Fault diagnosis}

After having built the bayesian network (qualitative step) and estimated the a priori probabilities of each node (quantitative step), the obtained graphical model is used to perform a fault diagnosis on the motor. For this application, the diagnosis consists in computing the a posteriori conditional probabilities according to new observations described in each one of the following scenarios.

Scenario 1: this scenario is related to the system's nominal operating mode. The bayesian network corresponding to this mode is given in figure 5 . In this case, given the fact that there is no observed fault on the system, the joint probability is equal to 1 . One can then compute for each node its own marginal probability value. According to the obtained results (see figure 5), we note that the probability for the rotation motion to be available is equal to $92.71 \%$ and that the stator, the rotor and the transmission shaft remain in their respective nominal state (ok mode), with probabilities equal to $99.49 \%, 95.92 \%$ and $98.88 \%$; respectively.

Scenario 2: in this second scenario we suppose that the rotation motion is unavailable $(P(M v t$ Rot $=n o)=1)$.

According to the conditional probabilities obtained by using BayesiaLab (figure 6), one can note that the fault

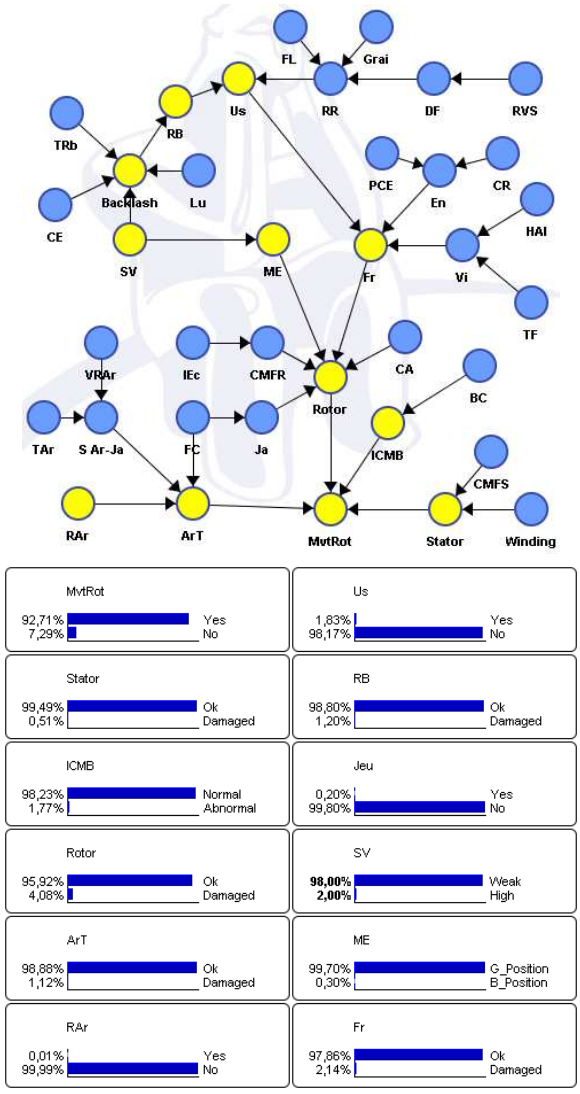

Fig. 5. Scenario 1: nominal operating mode

which is responsible of this abnormal operation can not be isolated with certainty. In fact, in absence of further information (measures, operator's observation, etc.) it is difficult to discriminate the origin of the dysfunction. However, we have the probability distributions on the participation of different possible causes in the occurrence of the observed fault. One tends then to believe that the unavailability of the rotation motion could have been caused by a fault on the rotor (with a probability value of $P($ rotor $=$ damaged $\mid M v t R o t=n o) \approx 0.56)$. This result is practically more plausible given the number of elements and components that are involved in its function and which can produce this faulty situation.

\begin{tabular}{|c|c|}
\hline 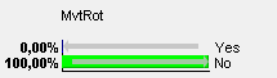 & $\begin{array}{l}\text { Us } \\
25,09 \% \Longleftarrow \text { Yes } \\
74,91 \% \Longleftarrow \text { No }\end{array}$ \\
\hline 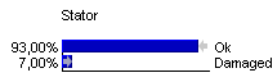 & $\begin{array}{l}\mathrm{RB} \\
89,64 \% \\
10,36 \% \text { — OK } \\
\text { Damaged }\end{array}$ \\
\hline $\begin{array}{l}\text { ICMB } \\
75,71 \% \\
24,29 \% \equiv \\
\end{array}$ & $\begin{array}{l}\text { Jeu } \\
1,83 \%: \\
98,17 \%\end{array}$ \\
\hline 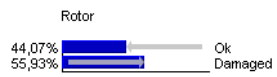 & $\begin{array}{l}\text { SV } \\
94,18 \% \text { Weak } \\
5,82 \% \square \text { Wigh }\end{array}$ \\
\hline $\begin{array}{l}\text { ArT } \\
84,64 \% \\
{ }_{15,36 \%}\end{array}$ & $\begin{aligned} & \text { ME } \\
& 95,91 \% \\
& 4,09 \% \text { G.Postion } \\
& \text { B-Postion }\end{aligned}$ \\
\hline $\begin{array}{c}\text { RAr } \\
0,14 \% \\
99,86 \% \\
\text { Nos } \\
\text { No }\end{array}$ & $\begin{array}{l}\mathrm{Fr} \\
{ }_{29,41 \%}^{70,49 \%} \rightleftharpoons \text { Damaged } \\
\end{array}$ \\
\hline
\end{tabular}

Fig. 6. Scenario 2: rotation motion unavailable 
Scenario 3: we consider the hypotheses of scenario 2 and, in addition to that, we suppose that there exists a sensor which measures the vibratory spectrum intensity (SV node on the graph of figure 3). In the case where strong vibrations are detected (which can be considered as observation or evidence), the probability of the node SV changes and becomes then a certitude: $P(S V=$ high $)=1$. According to this information, we do an update on the bayesian network of the system and calculate again all the a posteriori conditional probabilities (figure 7).

The results obtained by using BayesiaLab (figure 7) strengthen the belief on the fact that the rotor component would be the most probable cause of the observed malfunctioning situation. Indeed, strong vibrations can cause on the one hand degradation of the bearings and on the other hand a bad positioning of the equilibrium masses which can cause at their turn a degradation of the rotor. This justifies the increase in the probability value (from 0.56 to 0.87 ) that the rotor would be the cause of the unavailability of the rotation motion $(P($ rotor $=$ damaged $\mid$ MvtRot $=$ no, $S V=$ high) $\approx 0.87$ ).

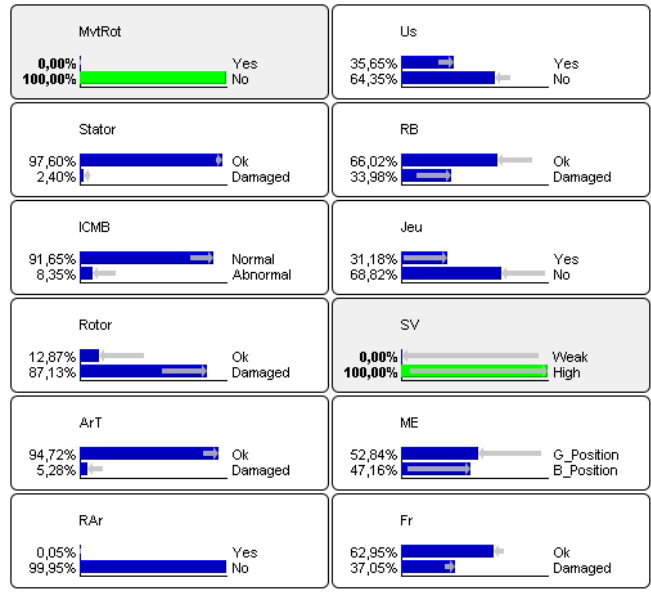

Fig. 7. Scenario 3: presence of sensors

\section{CONCLUSION}

In this paper, a bayesian based fault diagnosis method is presented. The use of this graphical and intuitive tool can be justified by the fact that, in practice, it is sometimes difficult to work with analytical model-based approaches, especially for complex systems, because of the difficulty to derive the corresponding model. Furthermore, for complex systems another factor has to be taken into account: the uncertainty. It is shown in the first part of this contribution how bayesian networks are used for fault diagnosis on a simple example related to a laptop starting problem. This method is then applied to diagnose faults on a real application: a permanent magnet synchronous motor. The construction of the graphical model of the system (identification of variables and their modes, causal relations, quantification of the probabilities, etc.) has been achieved according to the knowledge provided by an expert of the motor. Another way for deriving the model is by learning the structure and/or the parameters from a rich existing experimental or experience feedback database. This possibility has not been considered in this work because the studied motor was in fact at its design stage.
The availability of commercial as well as free softwares and toolboxes rend the use of bayesian networks more interesting. In our case, we have used the capabilities of the BayesiaLab software to simulate the different scenarios related to the rotation motion function of the transmission shaft. The analysis of the obtained results allowed to identify the vulnerable and critical components and thus, helped in designing and in planing appropriate maintenance tasks to perform in order to increase the system's availability and reliability.

\section{REFERENCES}

[1] R. Isermann. Supervision, Fault-Detection and FaultDiagnosis Methods - An introduction. Control Engineering Practice, volume 5, pages 639-652, 1997.

[2] R. Isermann. Model-based fault-detection and diagnosis - status and applications. Annual Reviews in Control, volume 29, pages 71-85, 2005.

[3] V. Venkatasubramanian, R. Rengaswamy and S.N. Kavuri. A review of process fault detection and diagnosis. Part II: Qualitative models and search strategies. Computers and Chemical Engineering, volume 27, issue 1, pages 313-326, 2003.

[4] V. Venkatasubramanian, R. Rengaswamy, K. Yin and S.N. Kavuri. A review of fault detection and diagnosis. Part III: Process history based methods. Computers and Chemical Engineering, volume 27, issue 1, pages 327-346, 2003.

[5] P. Naïm, P.H. Wuillemin, P. Leray, O. Pourret and A. Becker. Réseaux bayésiens. Eyrolles, 2004.

[6] P. Weber, M.-C. Suhner and B. Iung. System Approach-Based Bayesian Network to aid Maintenance of Manufacturing Process. In: Proceedings of the Sixth IFAC Symposium on Cost Oriented Automation (LowCost Automation 2001), october 8-9, Berlin, Germany.

[7] J. Pearl. Probabilistic reasoning in intelligent systems: Networks of plausible inference. Morgan Kaufmann Publishers, 1988.

[8] G. Cooper. Computational complexity of probabilistic inference using bayesian belief networks. Artificial Intelligence, volume 42, issues 2-3, pages 393-405, 1990.

[9] F.V. Jensen, S.L. Lauritzen, and K.G. Olesen. Bayesian updating in recursive graphical models by local computations. Computational Statistics Quarterly, volume 4, pages 269-282, 1990.

[10] M.I. Jordan, Z. Ghahramani, T. Jaakkola, and L.K. Saul. An introduction to variational methods for graphical models. Machine Learning, volume 37, issue 2, pages 183-233, 1999.

[11] G. Shafer and P. Shenoy. Local computation in hypertrees. Working Paper No 201, Business School, University of Kansas, 1988.

[12] R.D. Shachter, B. D'Ambrosio and B. A. Del Favero. Symbolic Probabilistic Inference in Belief Networks. in AAAI-90 conference, pages 126-131, 1990.

[13] L. Zhang and D. Poole. A simple approach to Bayesian Networks computations. In Proceeding of the Tenth Canadian Conference on Artificial Intelligence, Standford University, pages 171-178, 1994. 